\title{
Culex (Diptera: Culicidae) Mosquitoes in Jazan Region, Saudi Arabia, and Their Molecular Identification
}

\author{
Elsiddig Noureldin (D, Ommer Dafalla $(D$, Abdualziz Hakami, Mohammed Jubran, \\ Ahmed Alzhrani, Muhannad Mujally, Othman Shajari, Ali Khardali, and Zaki Eisa \\ Saudi Center for Disease Control and Prevention (SCDC), P.O. Box 716, Jazan 45142, Saudi Arabia \\ Correspondence should be addressed to Elsiddig Noureldin; elsiddigha@moh.gov.sa
}

Received 8 February 2021; Accepted 27 June 2021; Published 8 July 2021

Academic Editor: Marco Cucco

Copyright ( 92021 Elsiddig Noureldin et al. This is an open access article distributed under the Creative Commons Attribution License, which permits unrestricted use, distribution, and reproduction in any medium, provided the original work is properly cited.

\begin{abstract}
Morphological characteristics have been the gold standard method to identify mosquito species. However, morphological identification has many limitations including lack of expertise and damaging of external characters due to improper specimen handling. Therefore, we used the polymerase chain reaction technique (PCR) as an integrated tool to identify Culex mosquito species to establish a more precise and reliable identification system related to their spatial distribution in Jazan region. We identified Culex mosquito species and subspecies using taxonomic keys, and then we used the polymerase chain reaction technique (PCR) as an integrated tool to confirm and refine the list of Culex mosquito species in the region. Phylogenetic trees were constructed for the identified species, and their distinctive clustering was compared with their reference's species in the GenBank. We identified 7026 adult Culex mosquitoes belonging to 4 species. Culex tritaeniorhynchus was the predominant species (45\%), followed by Cx. quinquefasciatus (32\%), then Culex sitiens (20\%), and Cx. pipiens (3\%). The most infested areas by Culex in the region were Gizan and Sabya. The PCR achieved $100 \%$ success in identifying the four Culex mosquito species. We also report the molecular identification of $C x$. quinquefasciatus and $C x$. pipiens species for the first time in Jazan region while the molecular identification of $C x$. tritaeniorhynchus and $C x$. sitiens was reported for the first time in Jazan region and the whole Saudi Arabia. This study utilized for the first time PCR to identify Culex mosquito species in Jazan region. The PCR is a complementary and integrated taxonomy-based identification tool for mosquito species. This integration has the capacity to promote and enhance vector surveillance and control programs, as well as defining the genetic diversity of species in the region.
\end{abstract}

\section{Introduction}

Mosquito-borne diseases, i.e., malaria [1, 2], filariasis [3], Rift Valley fever [4, 5], West Nile virus [6], dengue, and others $[7,8]$, pose potential public health threats in Saudi Arabia.

Several authors have investigated the distribution of mosquito species in Saudi Arabia [1-16].

Most of these studies reported the predominance of Culex mosquitoes over other species. Within Culex species reported by these studies, Culex quinquefasciatus, Culex tritaeniorhynchus, and Cx. pipiens were the most dominant Culex mosquitoes in Saudi Arabia and Jazan region.

The abundance and distribution of mosquito fauna are influenced by many factors including, but not limited to, host availability, climatic conditions, especially rain and temperature, human mobility and activities, and land cover [17]. These factors have a potential impact on the vectorial capacity of mosquitoes for disease transmission.

The accurate identification of mosquito vector species and knowledge of their biology, ecology, and geographical distribution are considered important factors for surveillance and control of vectors and mosquito-borne diseases [18].

Morphological identification is the gold standard and the conventional method to identify mosquito species depending on their external characters [19].

However, morphological identification is highly time consuming and requires experienced taxonomists. 
Furthermore, improper handling of specimen damage is often occurred to some important characteristics such as bristles and scales leads to incomplete identification. Additionally, the similarity of shared morphological features by species complexes' members makes identification based on taxonomic keys alone a difficult task [20].

Polymerase chain reaction (PCR) is becoming a popular technique for mosquitoes identification based on their DNA sequencing and the fact that every species has its own genetic identity [19].

Molecular techniques to differentiate between $C x$. pipiens complex or/and other Culex species, or to differentiate between the $C x$. pipiens complex biotypes, are based on gel electrophoretic analysis of certain DNA fragments amplified by PCR [21]. PCR assay identifies $C x$. pipiens, $C x$. quinquefasciatus, and their hybrids based on the nucleotide sequence differences in the acetylcholinesterase gene Ace. 2 [22].

The aim of this study was to determine fauna of adult Culex mosquitoes (Diptera: Culicidae) and to produce precise and refine records of their species and their distribution in Jazan region based on morphological and genomic (molecular) identification. To the best of our knowledge, this is the first study in Jazan region to use molecular characteristics to identify Culex species and their genetic diversity.

\section{Materials and Methods}

2.1. Study Area. Jazan area is about $22,000 \mathrm{~km}^{2}$ and with 1.6 million population, lies between $16^{\circ}-12$ and $18^{\circ}-25$, latitude north, and located in the subtropical zone, southwestern of Saudi Arabia, with a coastal boundary of $250 \mathrm{~km}$ along the Red Sea and a $120 \mathrm{~km}$ border with the Republic of Yemen (Figure 1). This region includes over 3000 villages scattered along the area and about 100 islands located in the Red Sea, including the Farasan islands. It is surrounded by the Red Sea from the west and by Arabic Republic of Yemen from the south and east and Asir region from the north ([23]; GASTAT 2017: https://www.stats.gov.sa/en/5655).

2.2. Mosquito Collection. CDC miniature light traps were used for the adult mosquitos' collection from different parts of Jazan region from February 2018 to December 2019 (Table 1). Ten light traps were installed once per month in each of the houses, animals' shelters, wild vegetation, near wadies, sewerage plants, dams, and ponds from 1800 to $0600 \mathrm{hr}$.

For outdoor collections, a 2 kilogram block of dry ice (CO2) was wrapped in a Hessian bag above the trap. To minimize the mortality of the collected mosquitoes due to desiccation, damp cotton pads were kept in the collection cups. Collected mosquitoes were brought to the insectary of the Saudi Center for Disease Prevention and Control in Gizan city for morphological and genetic identification.

2.3. Mosquito Morphological Identification. Female Culex pipens and Culex quinquefasciatus mosquitoes were morphologically identified and differentiated by using wing measurements of intersection of costa, subcosta, and bifurcation of $R 2+3$ veins, as described by some taxonomic pictorial keys [24-26].

For the Females of Culex tritaeniorhynchus and Culex sitiens, relevant pictorial keys were used [24, 27, 28].

2.4. Molecular Identification of Culex Species. Previously identified Culex mosquitoes of individual species by morphometric methods were selected according to their location of capture.

DNA was extracted from the stored homogenate using GeneJET Genomic DNA Purification Kit (Thermo Scientific) following the manufacturer's recommendations: For every individual mosquito, legs were homogenized in a mortar and pestle (mini borosilicate glass chamber length $60 \mathrm{~mm}$ /pestle diameter $9.0 \mathrm{~mm}, 3.0 \mathrm{Ml}$, Fisherbrand) in $150 \mu \mathrm{L}$ of digestion solution and then the homogenate was transferred to 1.5 tubes. Twenty microliters of Proteinase $\mathrm{K}$ Solution was added and mixed thoroughly by vortexing to obtain a uniform suspension and incubated in the thermo shaker for 30 minutes at $56^{\circ} \mathrm{C}, 1300 \mathrm{rpm}$. Twenty microliters of RNase A Solution which was added and mixed by vortexing and incubated for $10 \mathrm{~min}$ at room temperature, then $200 \mu \mathrm{L}$ of lysis solution was added and mixed thoroughly by vortexing for $15 \mathrm{~s}$ until a homogeneous mixture is obtained. After adding $400 \mu \mathrm{L}$ of $50 \%$ ethanol and mixed by vortexing the prepared lysate was transferred to a GeneJET Genomic DNA Purification Column inserted in a collection tube and centrifuged for $1 \mathrm{~min}$ at $8000 \mathrm{rpm}$. The GeneJET Genomic DNA Purification Column was placed into a new $2 \mathrm{~mL}$ collection tube and $500 \mu \mathrm{L}$ of wash buffer 1 was added and centrifuged for $1 \mathrm{~min}$ at $8000 \mathrm{RPM}$, then the column was placed into a new collection tube and $500 \mu \mathrm{L}$ of wash buffer 2 was added and centrifuged for $3 \mathrm{~min}$ at 13,000 rpm. DNA Purification Column was transferred to a sterile $1.5 \mathrm{~mL}$ microcentrifuge tube, and $200 \mu \mathrm{L}$ of elution buffer was added to the center of the column membrane to elute genomic DNA. Incubated for $2 \mathrm{~min}$ at room temperature and centrifuged for $1 \mathrm{~min}$ at $8000 \mathrm{rpm}$.

The molecular identification of Culex species was performed by polymerase chain reaction technique (PCR) following the described procedure and using the primers provided in (Table 2.) $[22,29]$.

The PCR was carried out in a total volume of $50 \mu \mathrm{L}$ using Dream Tag Green Master Mix (Thermo Scientific) containing $25 \mu \mathrm{L}$ master mix ready to use, $1 \mu \mathrm{L}$ (10 pmol) of each reverse and species-specific forward primers, $5 \mu \mathrm{L}$ of DNA template, and $18 \mu \mathrm{L}$ nuclease-free water. The mix was subjected to initial denaturation at $94^{\circ} \mathrm{C}$ for 3 minutes, 30 cycles of denaturation $\left(94^{\circ} \mathrm{C}, 60 \mathrm{~s}\right)$, primer annealing $\left(51^{\circ} \mathrm{C}\right)$, primer extension $\left(72^{\circ} \mathrm{C}, 60 \mathrm{~s}\right)$, and final extension for 5 minutes. In each run, negative and positive controls were included. The PCR products amplifications were analyzed by gel electrophoresis (1.5 agarose in Tris-Acetate EDTA buffer) staining with ethidium bromide. The visualization was carried out using Gel Doc XR Imaging System (Bio-Rad).

Universal primers [30], LCO1490 and HCO2198 (Table 2), were used for the PCR amplification of a 710-bp fragment of the mitochondrial cytochrome oxidase subunit I 


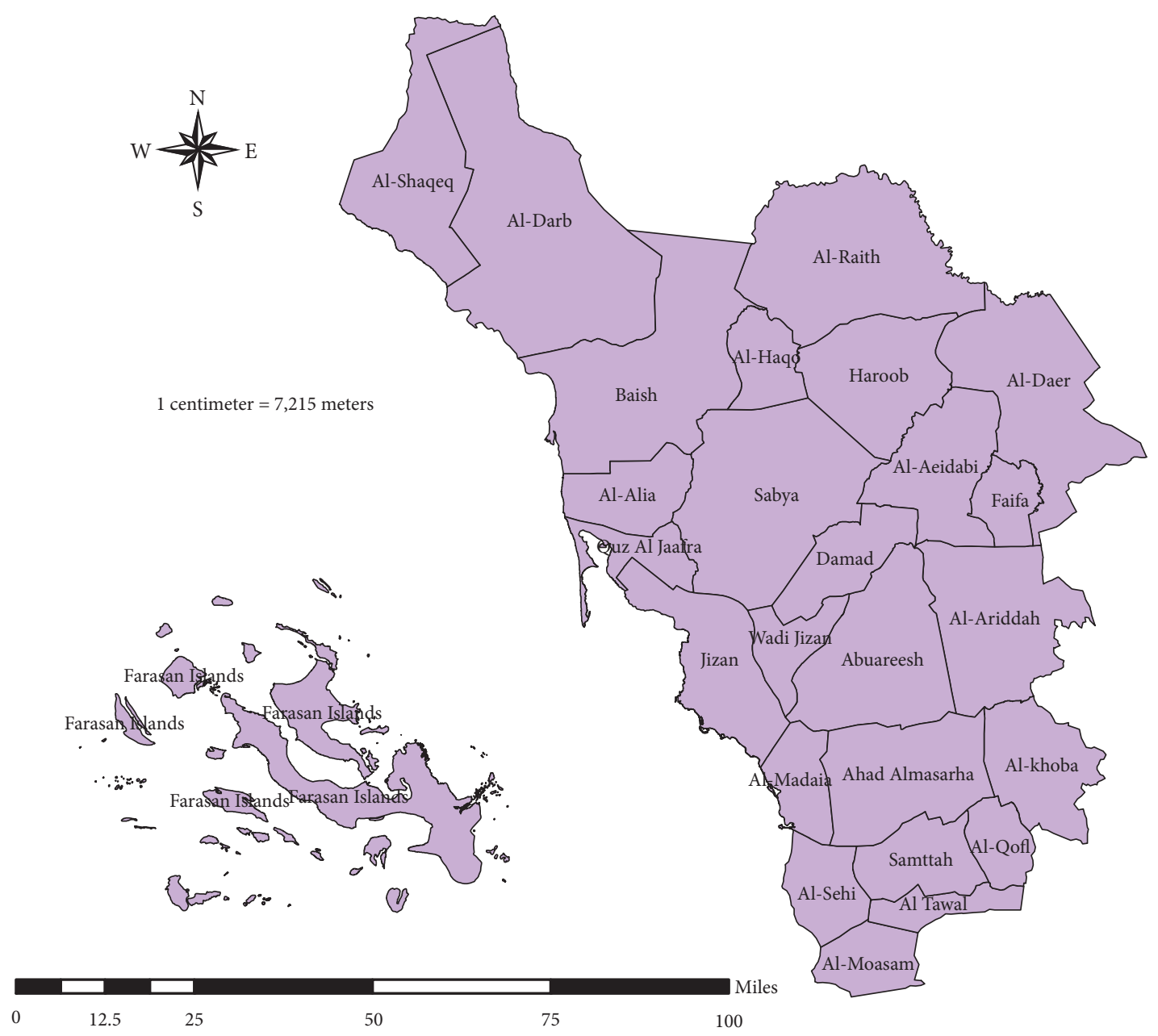

Figure 1: Boundaries of the different study areas in Jazan Region, southwest of Saudi Arabia.

gene (COI) for the samples not identified by the speciesspecific primers follow the same previous PCR procedure.

2.5. Sequencing. Purification and standard sequencing for PCR products were performed by Macrogen Company (Seoul, Korea). Sequencing reactions were performed in an ABI PRISM ${ }^{\circledR}$ 3730XL Analyzer (96 capillary type) using an ABI PRISM ${ }^{\circledR}$ BigDyeTM Terminator Cycle Sequencing Kit with AmpliTaq ${ }^{\circledR}$ DNA polymerase (FS enzyme) (Applied Biosystems), following the protocols supplied by the manufacturer. Single-pass sequencing was performed on each template using LCO1490 primer. Gel elution was performed using MG Gel Extraction SV (MD007) kit (MGmed), following the protocols supplied by the manufacturer.

The sequences were searched for sequence similarity through Basic local Alignment Search Tool (BLAST) (http:// www.ncbi.nlm.nih.gov/BLAST/) [31] and compared to reference sequences of Culex species detected in BLAST and downloaded from GenBank (http://www.ncbi.nlm.nih.gov/ genbank/).

Evolutionary relationship of taxa tree was constructed for each of the Culex species using the Molecular Evolutionary Genetic Analysis (MEGA 5 software).

\section{Results and Discussion}

Results of the identification and counts of Culex species mosquitoes are shown in Table 1. Seven thousand twenty-six adult Culex mosquitoes were collected from 25 different areas of Jazan region during 2018 and 2019 (Figure 1).

Clearly, the number of mosquito specimens was relatively low compared to the period of study (around 2 years). This is may be attributed to the fact that effective and extensive vector control activities were taken place during the last 5 years in the region to combat malaria, dengue, and rift valley, the most prevalent vector-borne diseases in the region.

In this study, four Culex species were present in the region, namely; Culex tritaeniorhynchus, $C x$. quinquefasciatus, $C x$. sitiens, and $C x$. pipiens. Their respective percentages of occurrence were $45 \%, 32 \%, 20 \%$, and $3 \%$.

Culex tritaeniorhynchus was the most abundant species encountered in 23 areas out of 25 , followed by $C x$. quinquefasciatus and $C x$. sitiens which occurred in 14 areas, and $C x$. pipiens in 4 areas.

These results are in accordance with the findings of $\mathrm{Al}$ Ahmad et al. [15] who indicated that Culex tritaeniorhynchus is a common widespread species in Saudi Arabia and has 
TABLE 1: Culex species in Jazan region.

\begin{tabular}{|c|c|c|c|c|c|}
\hline Area & Cx. tritaeniorhynchus & Cx. quinquefasciatus & Cx. pipiens & CX. sitiens & Total \\
\hline Gizan & 750 & 904 & 173 & 333 & 2160 \\
\hline Al-shegairy & 243 & 102 & 0 & 24 & 369 \\
\hline Ahad Almasarha & 0 & 0 & 0 & 29 & 29 \\
\hline Sabya (Qaem jaaferi) & 817 & 0 & 0 & 450 & 1267 \\
\hline Sabya (Wadi sabya) & 37 & 0 & 0 & 3 & 40 \\
\hline Samtah & 0 & 235 & 7 & 0 & 242 \\
\hline Damad & 53 & 0 & 0 & 204 & 257 \\
\hline Baish & 406 & 208 & 25 & 114 & 753 \\
\hline Aboareesh & 119 & 310 & 0 & 0 & 429 \\
\hline Al-tawal & 13 & 0 & 0 & 65 & 78 \\
\hline Al-madhaya & 229 & 0 & 0 & 0 & 229 \\
\hline Al-darb & 67 & 0 & 0 & 0 & 67 \\
\hline Al-ariddah (Batin asar) & 48 & 93 & 0 & 2 & 143 \\
\hline Haroob & 3 & 61 & 0 & 0 & 64 \\
\hline Al-shegaig & 84 & 0 & 0 & 0 & 84 \\
\hline Haroob (Wadi wasea) & 15 & 0 & 0 & 0 & 15 \\
\hline Faifa & 96 & 1 & 0 & 0 & 97 \\
\hline Al-gofl & 61 & 32 & 13 & 84 & 190 \\
\hline Al-dhabia & 0 & 54 & 0 & 2 & 56 \\
\hline Al-horath & 18 & 0 & 0 & 61 & 79 \\
\hline Al-sehi & 0 & 109 & 0 & 0 & 109 \\
\hline Al-aeidabi & 16 & 15 & 0 & 11 & 42 \\
\hline Al-aeidabi (Wadi qessi) & 25 & 0 & 0 & 9 & 34 \\
\hline Aiban & 8 & 18 & 0 & 0 & 26 \\
\hline Belgazzi & 12 & 103 & 0 & 0 & 115 \\
\hline Total & 3144 & 2266 & 218 & 1398 & 7026 \\
\hline$\%$ & 45 & 32 & 3 & 20 & \\
\hline
\end{tabular}

TABle 2: Primers used for molecular identification of Culex species.

\begin{tabular}{lc}
\hline Consensus CPI6 R & GCGGGTACCATGCTTAAATTTAGGGGTA \\
\hline Culex pipiens complex PQIO F & CCTATGTCCGCGTATACTA 698 bp \\
Cx. restuans R6 F & CCAAACACCGGTACCCAA 506 bp \\
Cx. salinarius S20 F & TGAGAATACATACCACTGCT 175 bp \\
\hline Cx. nigripalpus N90I F & ATACCCATGCGAAAGCATAC 404 bp \\
Cx. nigripalpus NRI080 R & GTACCGCGACCACACGACTT \\
\hline Cx. quinquefasciatus FCQ & GGT CGT ATC AAC AAA GTC CA 500 bp \\
Cx. quinquefasciatus RCQ & ATG GAT ACG GAC TAC ACT GG \\
\hline COI (LCO1490) & GGTCAACAAATCATAAAGATATTGG 740 bp \\
COI (HC02198) & TAAACTTCAGGGTGACCAAAAAATCA \\
\hline
\end{tabular}

been recorded from Jazan and other 14 provinces out of 15 . Similar results were also reported by Alsheikh et al. [32], who found $C x$. tritaeniorhynchus to be the most predominant species in the Red Sea coastal plain of Jazan region, followed by $C x$. quinquefasciatus. The findings are also in line with those of Al Ahmad et al.and Bakr et al. [14,33] who reported the same four Culex species from Jazan region. Culex tritaeniorhynchus, Cx. quinquefasciatus, and $C x$. sitiens have also been reported from different parts of Jazan region with the predominance of Culex tritaeniorhynchus over other Culex species [34].

In the present study, the most infested areas by Culex mosquitoes in Jazan region were Gizan (2160 specimens), Sabya (1267), Baish (753), and Aboareesh (429) (Table 1). This may be due to the nature of those areas, which are highly populated and relatively flat and permit for the formation of small stagnant water collections following rainfall and/or water pipe leakage in their urban areas [35]. The prevalence of the Culex species in those areas could be attributed also to their wide suitability to different breeding sites and variable extreme climatic factors prevailing there [11].

Three of the Culex species reported in the present study are considered important disease vectors, namely Culex tritaeniorhynchus, Cx. quinquefasciatus, and Cx. pipiens.

Characterizations of dominant mosquito vectors include well adaptation to a wide range of climatic conditions and habitats, high anthropophilic propensity, and variable adult resting behaviour [36].

Mosquito fauna and their distribution in Saudi Arabia had been widely investigated by many authors. For example, forty-nine mosquito species belonging to seven genera from 
1956 to 2017 (18 Anophelines and 31 Culicines) have been listed [37].

Out of the 31 Culicines, 19 species were belonging to the genus Culex Linnaeus (1758): one species under the subgenus Barraudius Edwards, 1921, Cx. (Barraudius) pusillus; one species under subgenus Culiciomyia Theobald, 1907, Cx. (Culiciomyia) nebulosus; one species under subgenus Oculeomyia Theobald, 1907, Cx. (Oculeomyia) bitaeniorhynchus; two species under subgenus Maillotia Theobald, 1907, Cx. (Maillotia) arbieeni and Cx. (Maillotia) salisburiensis; and fourteen species under subgenus Culex Linnaeus, 1758: $C x$. (Culex) decens, Cx. (Culex) duttoni, Cx. (Culex) laticinctus, $C x$. (Culex) mattinglyi, Cx. (Culex) mimeticus, Cx. (Culex) perexiguus, $C x$. (Culex) pipiens, $C x$. (Culex) quinquefasciatus, Cx. (Culex) simpsoni, Cx. (Culex) sinaiticus, Cx. (Culex) sitiens, $C x$. (Culex) theileri, Cx. (Culex) tritaeniorhynchus, and $C x$. (Culex) univittatus.

Likewise, 7 species of Culex had also been identified in Jazan region: (1) Culex pipiens from Gizan City, Alariddah, Baish, Ahad Almasarha, Samtah, Eldarab, Haroob, Bani Malik, and Farasan Islands; (2) Culex quinquefasciatus from Gizan City, Alariddah, Baish, Ahad Almasarha and Farasan Islands; (3) Culex tritaeniorhynchus from Gizan City, Alariddah, Baish, Ahad almasarha, Samtah, Eldarab, Haroob, and Bani Malik; (4) Culex sitiens from Gizan City, Alariddah, Baish, Ahad almasarha, Samtah, Wadi Jazan, and Farasan Islands; (5) Cx. Sinaiticus; (6) Cx. decens; (7) Cx. bitaeniorhynchus [33].

On the other hand, another 10 species have been identified from different areas of Jazan region including $C x$. tritaeniorhynchus, Cx. laticinctus, Cx. pipiens, Cx. quinquefasciatus, $C x$. sinaiticus, $C x$. simpsoni, $C x$. torrentium, $C x$. sitiens, Cx. univittatus, and Cx. tigripes [14].

Similarly, seven species were recorded from different parts of Jazan region including Culex tritaeniorhynchus, $C x$. quinquefasciatus, Cx. Sinaiticus, Cx. sitiens, Culex duttoni, Cx. Arbieeni, and Cx. mimeticus [34].

Cx. (Culex) perexiguus Theobald, 1903, and Cx. pusillus (Macquart) have been reported from the region $[24,38]$.

Consequently, 18 Culex species so far have been reported from Jazan region, namely: Culex tritaeniorhynchus, $C x$. laticinctus, Cx. pipiens, Cx. quinquefasciatus, Cx. sinaiticus, $C x$. univittatus, Cx. (Culex) perexiguus, Cx. pusillus, Cx. simpsoni, $C x$. torrentium, $C x$. sitiens, $C x$. decens, $C x$. bitaeniorhynchus, Cx. theileri, Cx. tigripes, Cx. duttoni, Cx. arbieeni, and $C x$. mimeticus [37].

Culex mosquitoes are proven or suspected vectors of West Nile, and Rift Valley fever viruses, microfilariae, and a variety of encephalitis $[39,40]$

It has been stated that $C x$. quinquefasciatus occurs more in the south of the Arabian Peninsula with isolated occurring of $C x$. pipiens in the southwestern mountains [41].

Due to the close similarity of $C x$. pipiens and $C x$. quinquefasciatus, many of their occurrences and morphological identifications in Saudi Arabia are problematic, especially females [37].

Considerable discrepancies in the larval and adult stages are exhibited between the two species, and it is difficult to distinguish them morphologically. Additionally, researchers working with Culex in Saudi Arabia have rarely, if not ever, used male genitalia diagnostic features to confirm identification of the two species [37].

This was the reason why we adopted the molecular technique, which is known to be highly specific and sensitive for the identification of Culex species complex and their hybrids [42, 43]. In addition, new methods of genomic (molecular) identification of species, subspecies, races, biotypes, and forms are more reliable and precise than older morphological identification methods of species complexes [44].

3.1. Culex quinquefasciatus (Figure 2). Larvae of Cx. quinquefasciatus breed in water bodies with a high degree of organic pollution and close to human habitats. Females are nocturnal and enter houses and bite the man in preference to other mammals [45].

CX. quinquefasciatus was found breeding all year round with peaks during winter in Taif, Jeddah, and Tabuk of Saudi Arabia [46]. In Jazan region, this species was collected from rocky pits and streams. It was found together with Anopheles arabiensis and $C x$. tritaeniorhynchus at the ratio of $1: 4: 1: 3$ [34].

The species is a primary vector of Wuchereria bancrofti, vector of avian malaria, western equine encephalomyelitis, and St. Louis encephalitis. It was implicated as a vector of dog heartworm [45, 47].

$C x$. quinquefasciatus and $C x$. pipiens, which are the main vectors of bancroftian filariasis, Wuchereria bancrofti worldwide including the Middle East countries, have been reported from the south-western districts of Saudi Arabia [6].

3.2. Culexpipiens (Figure 3). Larvae of Culex pipiens breed in various places ranging from highly polluted sewages and cesspits to clear pools and containers. It breeds in stagnant water in shaded or unshaded habitats. Females bite the man outdoors and indoors [41].

$C x$. pipiens larvae were reported all year round in Taif, Jeddah, and Tabuk. The peak was at autumn while high density was in summer when temperature is high [46].

$C x$. pipiens is a primary vector of periodic Bancroftian filariasis. It has been naturally infected with West Nile and Sindbis viruses in Israel, while it was infected with Rift Valley Fever virus and West Nile virus in Egypt [41].

$W$. bancrofti has been identified within foreign workers from five South-East Asian countries in Abha, southwest of the Saudi Arabia. Cx. pipiens may act as a potential vector of Bancroftian filariasis in Saudi Arabia [48].

Culex pipiens complex has occurred in different climatic and habitat zones, particularly temperate and temperatehumid regions of the world [44].

It is always difficult and problematic to differentiate morphologically between adults of $C x$. quinquefasciatus and Culex pipiens especially females. Nonetheless, taxonomists used to differentiate between the two species by wing venations where the intersection of the vein subcosta with costa is before the level of furcation of $R_{1+2}$ in the case of $C x$. 


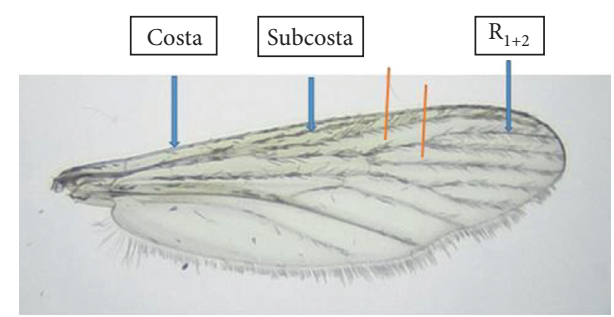

FIGURE 2: Wing of Culex quinquefasciatus showing the intersection of the vein subcosta with costa before the level of furcation of $R_{1+2}$.

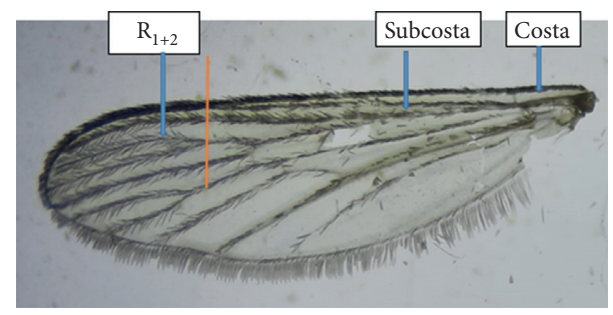

FIgURE 3: Wing of Culex pipiens showing the intersection of the vein subcosta with costa at the level of furcation of $R_{1+2}$.

quinquefasciatus (Figure 2), while this intersection is at or beyond the level of furcation of $R_{1+2}$ in the case of $C x$. pipiens (Figure 3) [24]. However, this is readily achieved by molecular characteristics.

Molecular identification of both $C x$. pipiens (Figures 4 and 5) and $C x$. quinquefasciatus (Figures 6 and 7) confirmed their initial morphological identification. Phylogenetic trees were constructed for $C x$. pipiens and $C x$. quinquefasciatus, and their distinctive clustering was compared with their reference's species in the GenBank as shown in Figures 5 and 7. It is worthy to mention that the molecular characteristics of $C x$. pipiens when compared with the records of the gene bank showed its similarity to those of Egypt, Kenya (100\%) and very close to those of Iran (99.85\%) (Table 3). While the molecular identification revealed one species of $C x$. quinquefasciatus that is similar to the Turkish species (100\%), and one subspecies of $C x$. pipiens quinquefasciatus ( $Q$ primers $500 \mathrm{bp}$ ) is similar to the Sri Lankan subspecies (100\%) (Table 3).

In Baljurashi Province of Saudi Arabia, the results of molecular identification of $C x$. pipiens using PCR technique revealed that the $C x$. pipiens cf-3 strain was the accurate definition after initial morphological identification of the species [49]. Likewise, the molecular characteristics of $C x$. quinquefasciatus samples from Yanbu Province of Saudi Arabia indicated that they were very close to those found in Pakistan, Brazil, and India (GenBank references; KF406862.1, MK575480.1, MH538709.1, and MH538707.1.) [50].

3.3. Culex tritaeniorhynchus (Figure 8(b)). The main morphological features found in Cx. tritaeniorhynchus are the presence of a median pale ring in the proboscis that extends proximally to the ventral surface of the proboscis (Figure 9(b)), and the fore and midfemora are entirely black

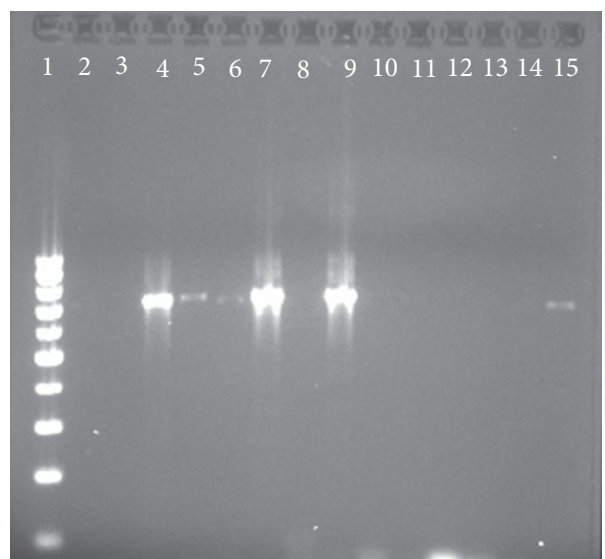

Figure 4: PCR amplified 700 bp DNA bands using consensus CPI6 $\mathrm{R}$ and $C x$. pipiens complex PQIO F primers. Lane 1: 100 bp ladder, lanes 2-15 tested samples.

(Figure 10(b)), whereas the furcation of $R_{2+3}$ is proximal to the furcation of $M_{1+2}$ (Figure 11(b)).

Larvae of $C x$ tritaeinorhynchus are found in many habitats that are sunlit and contain vegetation like the temporary, semi-permanent, and permanent ground water habitats, low-salinity tidal marshes, streams, ground pools, and swamps. Females feed primarily on pigs and cattle, but in their absence will feed on men [27]. It has been reported that larvae of $C x$. tritaeniorhynchus preferred aquatic habitats with wet muddy substrate and low total dissolved salts (TDS). Those areas are found in eastern Sarwat Mountain range near the cities in Ahd almasarha, Eleidabi, Sabya, AlAriddah, and Abuareesh [51].

Cx. tritaeniorhynchus was found in all types of breeding sites in the Red Sea coastal plain of Jazan region (man-made pools, especially turbid ones, rain pools, dams, rock pools, and domestic water tanks). It was also noticed to share breeding sites with Anopheles arabiensis, the main malaria vector in the region [23].

Cx. tritaeinorhynchus is a primary vector of Japanese B encephalitis in the oriental region [27] and Rift valley virus in Jazan region of Saudi Arabia with a biting preference for humans and sheep [4]. In the present study, the molecular identification of $C x$. tritaeniorhynchus using the universal primer COI (Figure 12), along with the constructed phylogenetic trees and their distinctive clustering, was compared with their reference's species in the GenBank (Figure 13) and showed that it is quite similar to the species of USA, Japan, and China (100\% similarity, Table 3).

3.4. Culex sitiens (Figure 8(a)). The main morphological features of the proboscis indicate that the median pale ring is present, but not extending proximally to the ventral surface as in Cx. tritaeinorhynchus (Figure 9(a)); the fore and midfemora are speckled (Figure 10(a)). On the other hand, the furcation of $R_{2+3}$ is distal to the furcation of $M_{1+2}$ (Figure 11(a)).

Bionomics of $C x$. sitiens showed various breeding habitats including fresh ground water, salt, brackish, and 


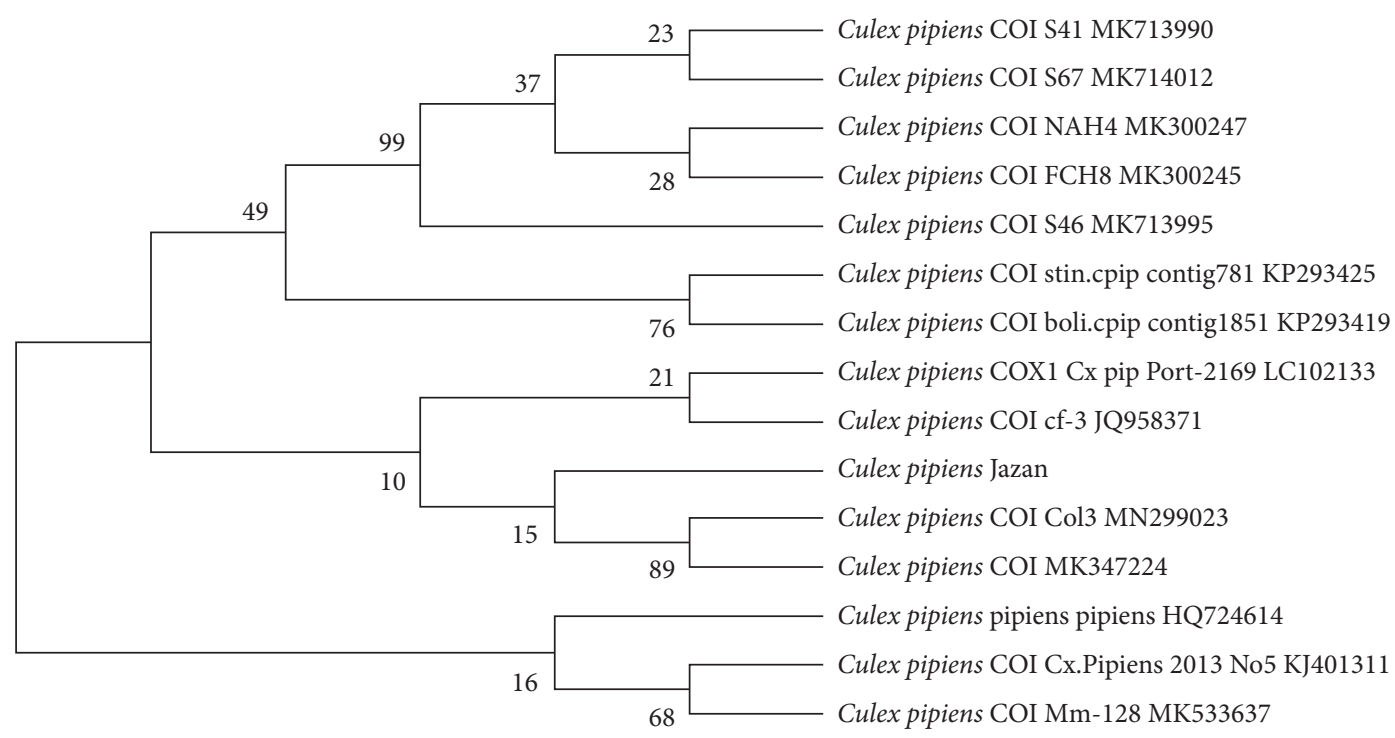

FIgURE 5: Evolutionary relationship of taxa of Culex pipiens identified in Jazan region using MEGA 5.

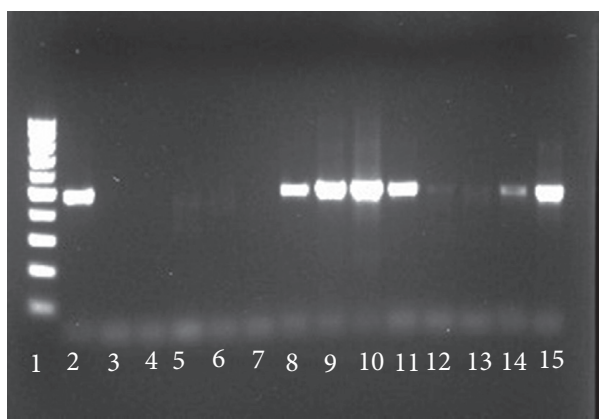

Figure 6: PCR amplified 500 bp DNA bands using Cx. quinquefasciatus specific primers. Lane 1: 100 bp ladder, lane 2: positive control, lane 3: negative control, lane 4: negative sample, lanes 5-15 positive samples.

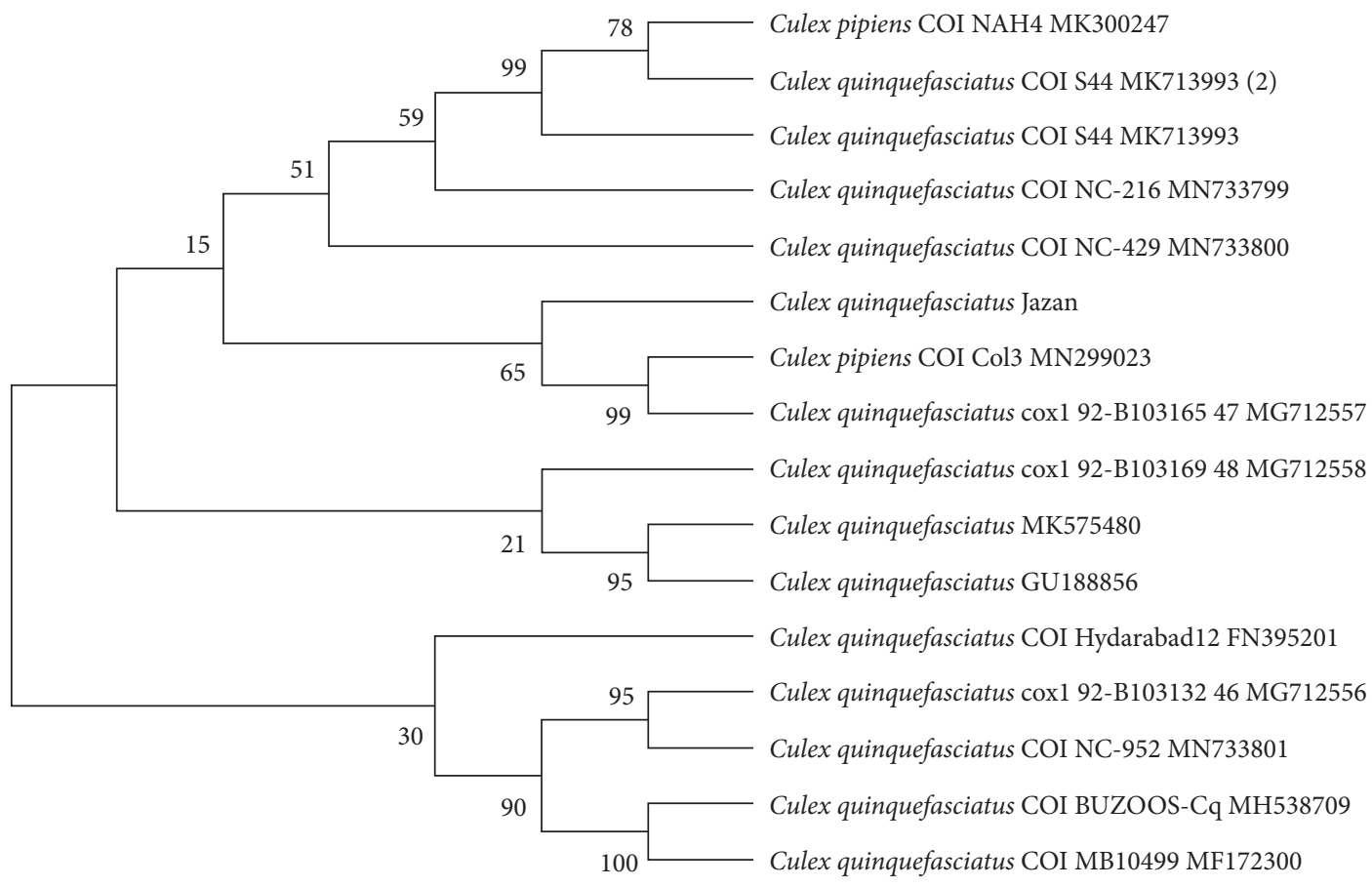

FIgURE 7: Evolutionary relationship of taxa of Culex quinquefasciatus identified in Jazan region using MEGA 5. 
TABLE 3: Similarity of Culex species in Jazan Region related to gene bank references.

\begin{tabular}{|c|c|c|c|}
\hline Culex species & Gene bank accession no. & Similarity (\%) & Country \\
\hline \multirow{3}{*}{ Culex tritaeniorhynchus } & KJ012245.1 & 100 & USA \\
\hline & AB738247.1 & 100 & Japan \\
\hline & MF179221 & 100 & China \\
\hline \multirow{3}{*}{ Culex pipiens } & MT199095 & 100 & Egypt \\
\hline & MK300250 & 100 & Kenya \\
\hline & JQ958371 & 99.85 & Iran \\
\hline Culex quinquefasciatus & MK713993.1 & 100 & Turkey \\
\hline Culex pipiens quinquefasciatus (Q primers $500 \mathrm{bp}$ ) & AF089002.1 & 100 & Sri Lanka \\
\hline \multirow{3}{*}{ Culex sitiens } & MN552296 & 100 & Guinea \\
\hline & MK300241 & 100 & Kenya \\
\hline & MF179212 & 100 & China \\
\hline
\end{tabular}

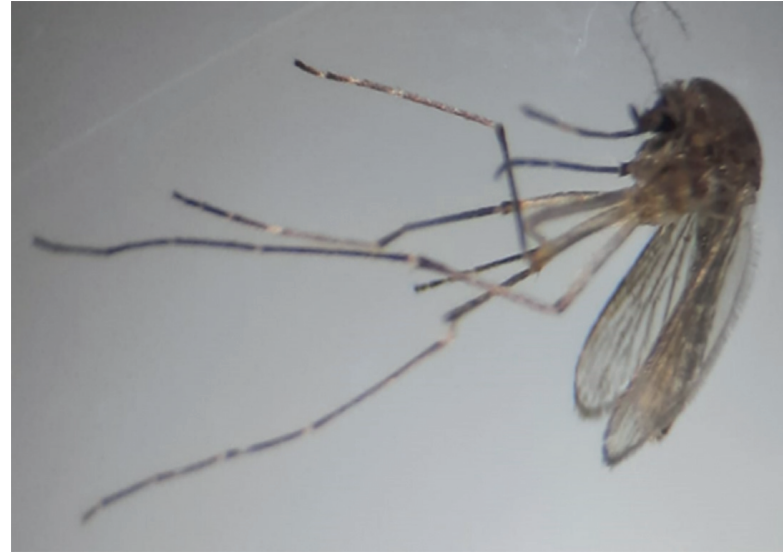

(a)

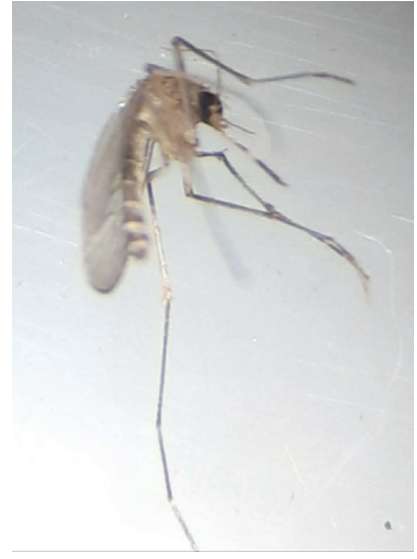

(b)

Figure 8: Culex sitiens (a) and Culex tritaeniorhynchus (b).

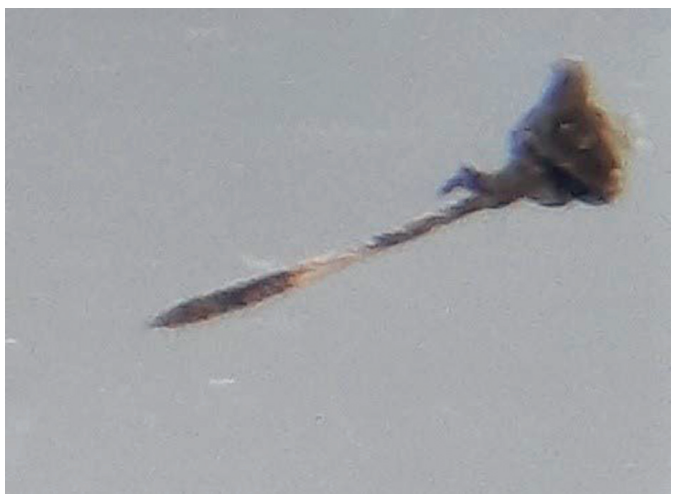

(a)

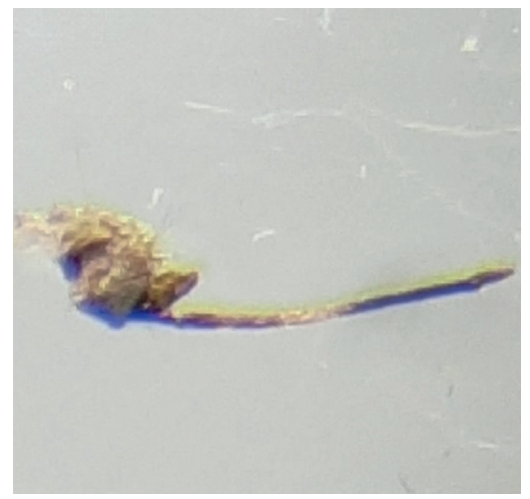

(b)

Figure 9: Proboscis of Culex sitiens (a) and Culex tritaeniorhynchus (b).

artificial containers in coastal, urban, and suburban areas [41].

It can also be found in almost every stagnant brackish waters with full sunlight [52]. Females feed primarily on pigs and birds, but will also bite men. It is an implicated vector of Japanese B encephalitis [41].

In Jeddah, Saudi Arabia, the density of $C x$. sitiens was directly related to temperature. Its peak of density is in 


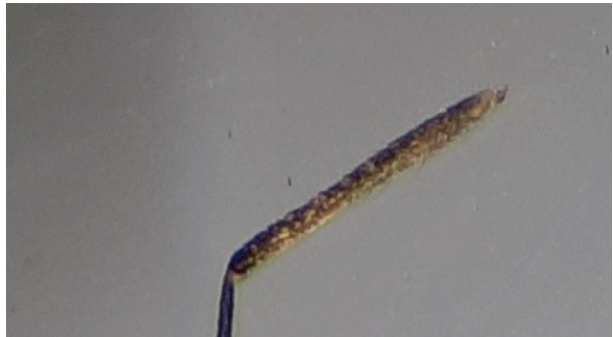

(a)

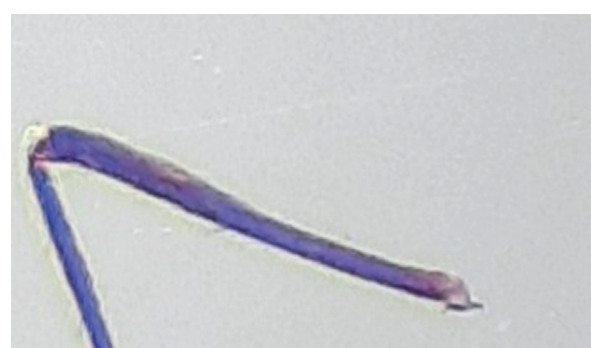

(b)

FIgURe 10: Fore and midfemora of Cx. sitiens (speckled) (a) and (entirely black) Cx. tritaeniorhynchus (b).

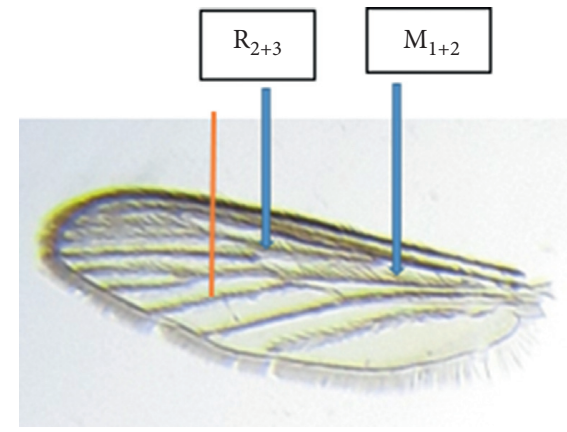

(a)

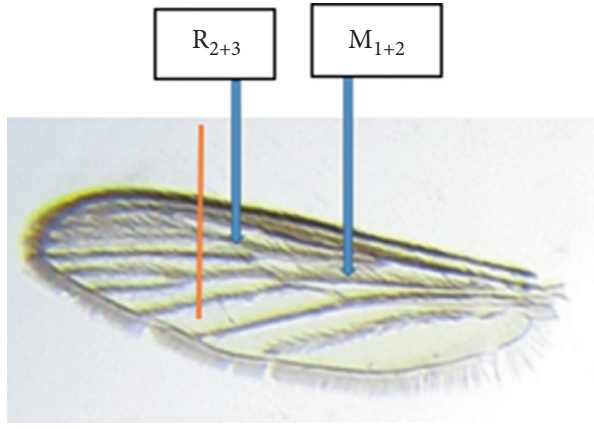

(b)

FIgURE 11: Furcation of $R_{2+3}$ of $C x$. sitiens (distal to furcation of $M_{1+2}$ ) (a) and (proximal to furcation of $M_{1+2}$ ) Cx. tritaeniorhynchus (b).

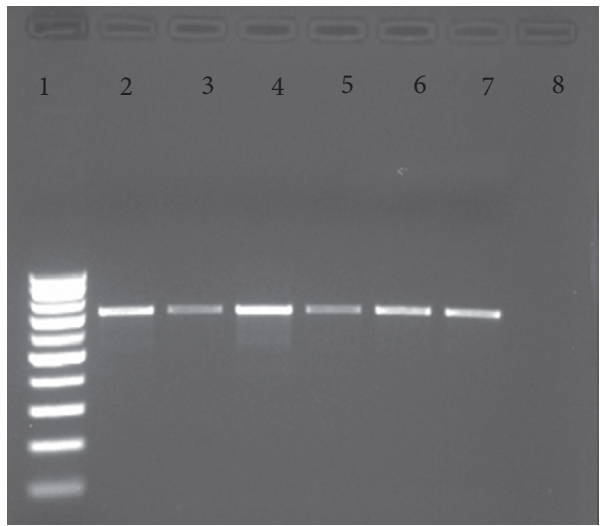

FIGURE 12: PCR amplified 745 bp DNA bands using COI primers to identify Culex tritaeniorhynchus and Culex sitiens. Lane 1: 100 bp ladder, lane 2: positive control, lanes 3-7: tested samples, lane 8: negative control.

summer, and it is highly abundant in spring, while it breeds throughout the year [46].

Cx. sitiens molecular identification in the present study using the universal primer COI (Figure 12), along with the constructed phylogenetic trees and their distinctive clustering, when compared with their reference's species in the GenBank (Figure 14) revealed that it is similar to the African species of Guinea and Kenya (100\%) and China (100\%) (Table 3).

All mosquito species identified morphologically in this study have also been molecularly identified, assuring absolute compatibility between taxonomic and molecular identification, confirming that PCR is a complementary tool for the identification of mosquito species. 


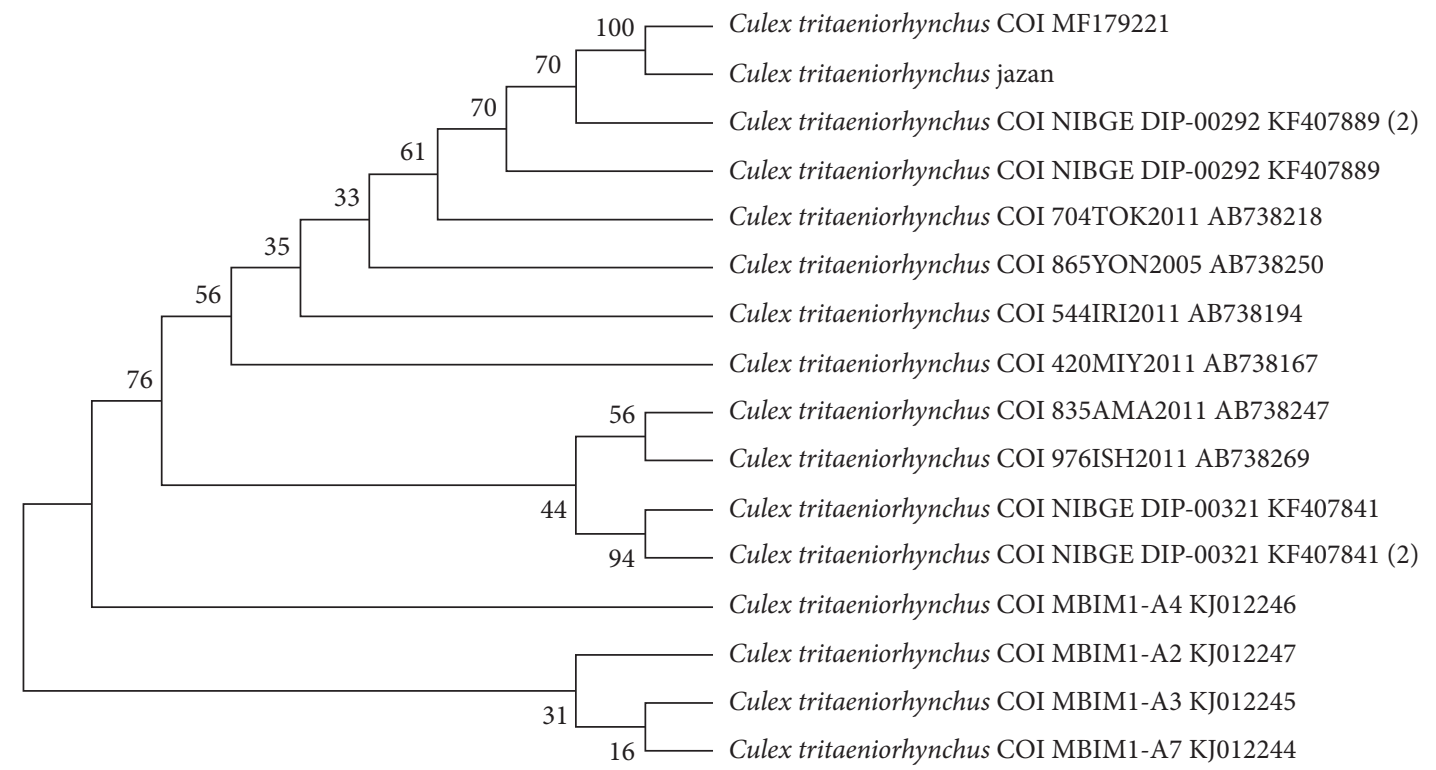

FIgURE 13: Evolutionary relationship of taxa of Culex tritaeniorhynchus identified in Jazan region using MEGA 5.

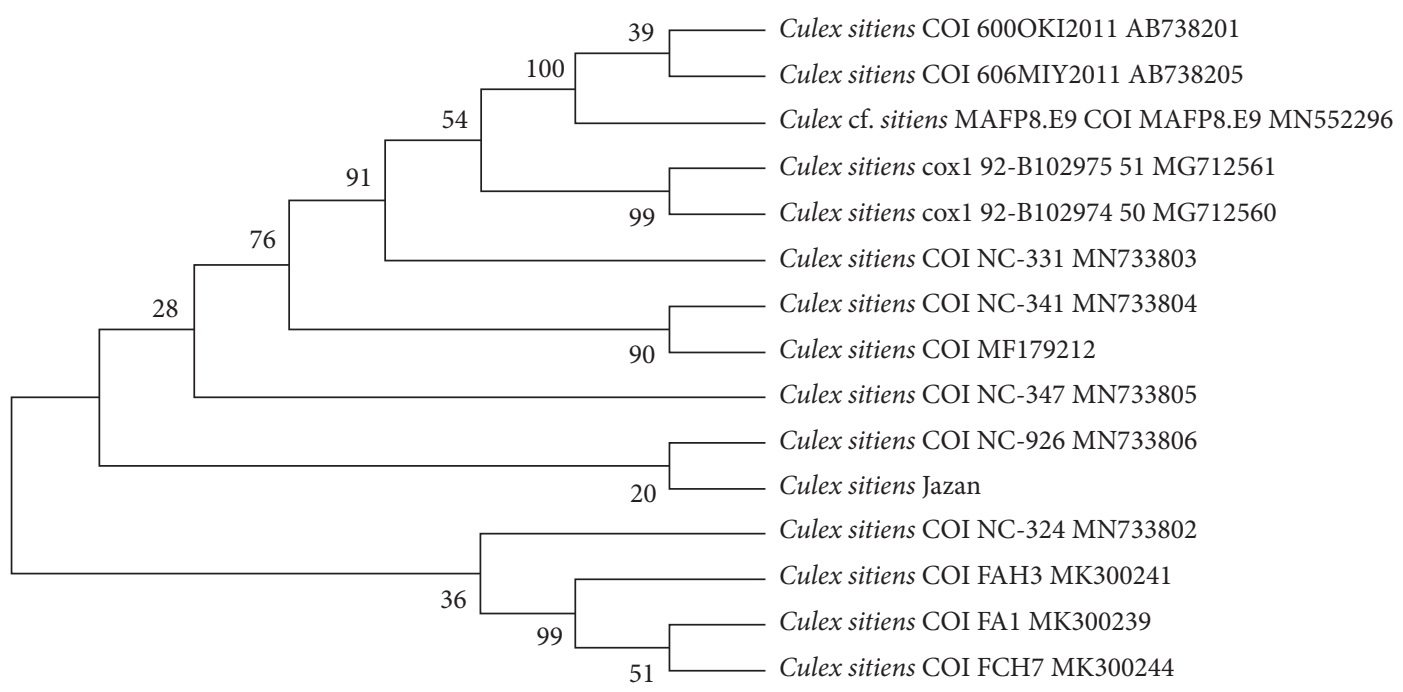

FIGURE 14: Evolutionary relationship of taxa of Culex sitiens identified in Jazan region using MEGA 5.

\section{Conclusion}

In this study, Culex tritaeniorhynchus was the predominant species in Jazan region, followed by $C x$. quinquefasciatus, then $C x$. sitiens, and $C x$. pipiens. The most infested areas by Culex mosquitoes in the region were Gizan and Sabya.

The results showed the potentiality of integrating the morphological and molecular identification to refine the list of Culex mosquito species and accurately document their geographical distribution in the Jazan region. This integration has the capacity to promote and enhance vector surveillance and control programmes, as well as defining the genetic diversity of species in the region.

\section{Data Availability}

The data used in this study are included within the article.

\section{Conflicts of Interest}

The authors declare that they have no conflicts of interest.

\section{References}

[1] D. A. Warrell, "Leishmaniasis, malaria and schistosomiasis in Saudi Arabia," Saudi Medical Journal, vol. 14, pp. 203-208, 1993.

[2] A. M. Abdoon and A. M. Alshahrani, "Prevalence and distribution of anopheline mosquitoes in malaria endemic areas of Asir region, Saudi Arabia," Eastern Mediterranean Health Journal, vol. 9, no. 3, pp. 240-247, 2003.

[3] F. Hawking, The Distribution of Human Filariases throughout the World, Mimeograph WHO/FIL/73.114, 1973.

[4] P. G. Jupp, A. Kemp, A. Grobbelaar et al., "The 2000 epidemic of Rift Valley fever in Saudi Arabia: mosquito vector studies," 
Medical and Veterinary Entomology, vol. 16, no. 3, pp. 245252, 2002.

[5] M. Al-Hazmi, E. A. Ayoola, M. Abdurahman et al., "Epidemic Rift Valley fever in Saudi Arabia: a clinical study of severe illness in humans," Clinical Infectious Diseases, vol. 36, no. 3, pp. 245-252, 2003.

[6] K. H. Al Ali, A. A. El-Badry, M. Al Ali, W. S. M. El-Sayed, and H. A. El-Beshbishy, "Phylogenetic analysis of Aedes aegypti based on mitochondrial ND4 gene sequences in Almadinah, Saudi Arabia," Iranian Journal of Biotechnology, vol. 14, Article ID e1329, 2016.

[7] M. Fakeeh and A. M. Zaki, "Virologic and serologic surveillance for dengue fever in Jeddah, Saudi Arabia, 1994-1999," The American Journal of Tropical Medicine and Hygiene, vol. 65, no. 6, pp. 764-767, 2001.

[8] N. A. Khan, E. I. Azhar, S. El-Fiky et al., "Clinical profile and outcome of hospitalized patients during first outbreak of dengue in Makkah, Saudi Arabia," Acta Tropica, vol. 105, no. 1, pp. 39-44, 2008.

[9] M. Fakeeh and A. M. Zaki, "Dengue in Jeddah, Saudi Arabia, 1994-2002," Dengue Bulletin, vol. 27, pp. 13-18, 2003.

[10] P. F. Mattingly and K. L. Knight, "The mosquito of Arabia I," Bulletin of the British Museum (Natural History), vol. 4, no. 3, pp. 91-141, 1956.

[11] M. A. R. Abdullah and A. I. Merdan, "Distribution and ecology of the mosquito fauna in the southwestern Saudi Arabia," Journal of the Egyptian Society of Parsitology, vol. 25, no. 3, pp. 815-837, 1995.

[12] B. R. Miller, M. S. Godsey, M. B. Crabtree et al., "Isolation and genetic characterization of Rift Valley fever virus from Aedes vexans arabiensis, Kingdom of Saudi Arabia," Emerging Infectious Diseases, vol. 8, no. 12, pp. 1492-1494, 2002.

[13] A. M. Alahmed, M. A. Al Kuriji, S. M. Kheir, S. A. Alahmedi, M. J. Al Hatabbi, and M. A. Al Gashmari, "Mosquito fauna (Diptera: Culicidae) and seasonal activity in Makkah Al Mukarramah Region, Saudi Arabia," Journal of the Egyptian Society of Parasitology, vol. 39, pp. 991-1013, 2009.

[14] A. M. Alahmed, M. A. Al Kuriji, S. M. Kheir, H. M. Al Haqawi, and E. A. A. Sultan, "Mosquito (Diptera: Culicidae) and their seasonal activity in Jazan Region, Saudi Arabia," Journal of the Saudi Society of Agricultural Sciences, vol. 9, pp. 136-138, 2010.

[15] A. M. Al Ahmad, M. F. Sallam, M. A. Khuriji, S. M. Kheir, and S. Azari-Hamidian, "Checklist and pictorial key to fourthinstar larvae of mosquitoes (Diptera: Culicidae) of Saudi Arabia," Journal of Medical Entomology, vol. 48, no. 4, pp. 717-737, 2011.

[16] K. Al-Ghamdi, M. Alikhan, J. Mahyoub, and Z. I. Afifi, "Studies on identification and population dynamics of anopheline mosquitoes from Jeddah Province of Saudi Arabia," Bioscience Biotechnology Research Communications, vol. 1, pp. 19-24, 2008.

[17] L. Delgado-Petrocelli, A. Camardiel, V. H. Aguilar, N. Martinez, K. Córdova, and S. Ramos, "Geospatial tools for the identification of a malaria corridor in Estado Sucre, a Venezuelan north-eastern state," Geospatial Health, vol. 5, no. 2, pp. 169-176, 2011.

[18] M. Amini, A. A. Hanafi-Bojd, A. A. Aghapour, and A. R. Chavshin, "Larval habitats and species diversity of mosquitoes (Diptera: Culicidae) in West Azerbaijan Province, Northwestern Iran," BMC Ecology, vol. 20, no. 1, p. 60, 2020.

[19] A. Chan, L.-P. Chiang, H. C. Hapuarachchi et al., "DNA barcoding: complementing morphological identification of mosquito species in Singapore," Parasites \& Vectors, vol. 7, no. $1,2014$.

[20] U. Jinbo, T. Kato, and M. Ito, "Current progress in DNA barcoding and future implications for entomology," Entomological Science, vol. 14, no. 2, pp. 107-124, 2011.

[21] N. Shahhosseini, M. H. Kayedi, M. M. Sedaghat, T. Racine, G. P. Kobinger, and S. H. Moosa-Kazemi, "DNA barcodes corroborating identification of mosquito species and multiplex real-time PCR differentiating Culex pipiens complex and Culex torrentium in Iran," PloS One, vol. 13, no. 11, Article ID e0207308, 2018.

[22] A. Stephen, M. B. Crabtree, and M. S. Harry, "Polymerase chain reaction assay identifies culex nigripalpus: part of an assay for molecular identification of the common culex (culex mosquitoes of the eastern United States," Journal of the American Mosquito Control Association, vol. 19, no. 2, pp. 115-120, 2003.

[23] A. A. Alsheikh, "Larval habitat, ecology, seasonal abundance and vectorial role in malaria transmission of Anopheles arabiensis in Jazan Region of Saudi Arabia," Journal of the Egyptian Society of Parasitology, vol. 41, no. 3, pp. 615-634, 2011 Dec.

[24] R. E. Harbach, "Pictorial keys to the genera of mosquitoes, subgenera of Culex and the species of Culex (Culex) occurring in southwestern Asia and Egypt, with a note on the subgeneric placement of Culex deserticola (Diptera: Culicidae)," Mosquito Systematics, vol. 17, pp. 83-107, 1985.

[25] S. Antunes-de Morais, C. Moratore, L. Suesdek, and M. T. Marrelli, "Genetic-morphometric variations in culex quinquefaciatus from Brazil and La Plata, Argentina," Memórias do Instituto Oswaldo Cruz, vol. 105, no. 5, 2010.

[26] H. Dengham, S. H. Moosa-Kazemi, J. Sadraei, and H. Soleimani, "The ecological aspects of Culex pipens (Diptera: Culicidae) in central Iran," Journal of Arthropod-Borne Diseases, vol. 8, no. 1, pp. 35-42, 2014.

[27] R. A. Bram, "Contributions to the mosquito fauna of Southeast Asia-II. The genus Culex in Thailand (Diptera: Culicidae)," Contributions of the American Entomological Institute, vol. 2, no. 1, pp. 1-296, 1967.

[28] B. P. Das, "Pictorial key to common species of Culex (Culex) mosquitoes associated with Japanese encephalitis virus in India," in Mosquito Vectors of Japanese Encephalitis Virus from Northern India, pp. 25-42, Springer India, New Delhi, India, 2013.

[29] U. P. K. Hettiaratchi, D. H. N. Munasingha, N. V. Chandrasekharan, E. H. Karunanayake, and N. Jayasekera, "A polymerase chain reaction based method for the detection of Culex quinquefasciatus (Diptera: Culicidae)," Bulletin of Entomological Research, vol. 90, no. 1, pp. 63-68, 2000.

[30] O. Folmer, M. Black, W. Hoeh, R. Lutz, and R. Vrijenhoek, "DNA primers for amplification of mitochondrial cytochrome $\mathrm{c}$ oxidase subunit I from diverse metazoan invertebrates," Molecular Marine Biology and Biotechnology, vol. 3, no. 5, pp. 294-299, 1994.

[31] S. F. Altschul, T. L. Madden, A. A. Schäffer et al., "Gapped BLAST and PSI-BLAST: a new generation of protein database Search programs," Nucleic Acids Research, vol. 25, pp. 33893402, 1997.

[32] A. A. Alsheikh, M. H. Zafer, A. G. Anaami et al., "Potential mosquito vectors of arboviral diseases in Jazan Region, Saudi Arabia," Bioscience Biotechnology Research Communications, vol. 6 , no. 2, pp. 142-149, 2013. 
[33] R. Bakr, M. Nassar, N. El-Barky, T. Kotb, H. Badrawy, and M. Abdeldayem, "Prevalence of mosquitoes in Jazan Province, Saudi Arabia," Egyptian Academic Journal of Biological Sciences. A, Entomology, vol. 7, no. 2, pp. 15-27, 2014.

[34] E. I. Khater, M. M. Sowilem, M. F. Sallam, and A. M. Alahmed, "Ecology and habitat characterization of mosquitoes in Saudi Arabia," Tropical Biomedicine, vol. 30, no. 3, pp. 409-427, 2013.

[35] T. A. Al-Azraqi, A. A. El Mekki, and A. A. Mahfouz, "Seroprevalence of dengue virus infection in Aseer and Jizan regions, Southwestern Saudi Arabia," Transactions of The Royal Society of Tropical Medicine and Hygiene, vol. 107, no. 6, pp. 368-371, 2013.

[36] M. E. Sinka, Y. Rubio-Palis, S. Manguin et al., "The dominant Anopheles vectors of human malaria in the Americas: occurrence data, distribution maps and bionomic précis," Parasites Vectors, vol. 3, 2010.

[37] A. M. Alahmed, K. Munawar, S. M. S. Khalil, R. E. Harbach, and R. E. Harbach, "Assessment and an updated list of the mosquitoes of Saudi Arabia," Parasites and Vectors, vol. 12, no. 1, p. 356, 2019.

[38] T. V. Gaffigan, R. C. Wilkerson, J. E. Pecor, J. A. Stoffer, and T. Anderson, Systematic Catalog of Culicidae Suitland, Walter Reed Biosystematics Unit, Museum Support Center, Smithsonian Institution, Suitland, MD, USA, 2019, http://www. mosquitocatalog.org/.

[39] J. T. Michael, "Members of the Culex pipiens complex as vectors of viruses," Journal of the American Mosquito Control Association, vol. 28, no. 4s, pp. 123-126, 2012.

[40] S. R. Irish, H. M. Al-Amin, H. N. Paulin et al., "Molecular xenomonitoring for Wuchereria bancrofti in Culex quinquefasciatus in two districts in Bangladesh supports transmission assessment survey findings," PLOS Neglected Tropical Diseases, vol. 12, no. 7, Article ID e0006574, 2018.

[41] R. E. Harbach, "The mosquitoes of the subgenus Culex in southwestern Asia and Egypt (Diptera: Culicidae)," Contributions of the American Entomological Institute, vol. 24, no. 1, pp. 1-236, 1988.

[42] A. Diaz-Badillo, B. G. Bolling, G. Perez-Ramirez et al., "The distribution of potential West Nile virus vectors, Culex pipiens pipiens and Culex pipiens quinquefasciatus (Diptera: Culicidae), in Mexico City," Parasites and Vectors, vol. 4, no. 1, p. 70, 2011.

[43] B. O. Motayo, B. A. Onoja, A. O. Faneye, and J. A. Adeniji, "Seasonal abundance and molecular identification of West Nile virus vectors, Culex pipens and Culex quinquefasciatus (diptera: culicidae) in Abeokuta, South-West, Nigeria," African Health Sciences, vol. 16, no. 1, pp. 135-140, 2016.

[44] M. H. Kayedi, F. Sepahvand, E. Mostafavi et al., "Morphological and molecular identification of Culicidae mosquitoes (Diptera: Culicidae) in Lorestan province, Western Iran," Heliyon, vol. 6, no. 8, Article ID e04480, 2020.

[45] S. Sirivanakarn, "Medical entomology studies-III. A revision of the subgenus Culex in the Oriental region (Diptera: Culicidae)," Contributions of the American Entomological Institute, vol. 12, no. 2, pp. 1-272, 1976.

[46] M. I. Hassan, H. A. Al Ashry, M. Shobrak, and M. A. Kenawy, "Seasonal abundance of the common mosquitoes: Culex pipiens, Cx. quinquefasciatus and Cx. sitiens (Diptera: Culicidae) in the western coast of Saudi Arabia," Egyptian Academic Journal of Biological Sciences E Medical Entomology and Parasitology, vol. 8, no. 1, pp. 13-22, 2016.
[47] S. J. Carpenter and W. J. LaCasse, Mosquitoes of North America (North of Mexico), University of California Press, Berkeley, Los Angeles, 1955.

[48] M. S. Omar, "A survey of bancroftian filariasis among South East Asian expatriate workers in Saudi Arabia," Tropical Medicine and International Health, vol. 1, no. 2, pp. 155-160, 1996.

[49] T. S. Alghamdi, Study of the Seasonal Fluctuation of Mosquitoes Population with Evaluation of the Effectiveness of the Insecticides Used against the Dominant Species in Baljurashi Province, Ph.D. Thesis, Faculty of Sciences King Abdulaziz University, Jeddah, Saudi Arabia, 2018.

[50] S. T. Alerwi, N. Alkenani, J. A. Mahyoub, A. Alghamdi, S. A. Hanan, Y. Anwar et al., "Molecular identification and bio-control of Culex quinquefasciatus from Yanbu region," Journal of Entomology and Zoology Studies, vol. 7, no. 6, pp. 1081-1086, 2019.

[51] M. F. Sallam, A. M. Al Ahmed, M. S. Abdel-Dayem, and M. A. R. Abdullah, "Ecological niche modeling and land cover risk areas for rift valley fever vector, Culex tritaeniorhynchus giles in jazan, Saudi Arabia," PLoS One, vol. 8, no. 6, Article ID e65786, 2013.

[52] S. Prummongkol, C. Panasoponkul, C. Apiwathnasorn, and U. Lek-Uthai, "Biology of Culex sitiens, a predominant mosquito in Phang Nga, Thailand after a tsunami," Journal of Insect Science, vol. 12, pp. 1-8, 2012. 